\title{
Comparing and contrasting knowledge of pressure ulcer assessment, prevention and management in people with spinal cord injury among nursing staff working in two metropolitan spinal units and rehabilitation medicine training specialists in a three-way comparison
}

\author{
N Gupta ${ }^{1}$, B Loong ${ }^{2}$ and G Leong ${ }^{1,3}$ \\ ${ }^{1}$ Spinal Cord Injuries Unit, Royal North Shore Hospital, St Leonards, New South Wales, Australia; ${ }^{2}$ Harvard University Statistics \\ Department, Cambridge, MA, USA and ${ }^{3}$ Statewide Spinal Outreach Service, Royal Rehabilitation Centre, Ryde, Sydney, Australia
}

\begin{abstract}
Aim: To assess for differences in knowledge of pressure ulcer (PU) prevention and management among nurses working in two metropolitan spinal cord injury $(\mathrm{SCl})$ units, and between nurses and rehabilitation registrars (doctors).

Background: There is anecdotal evidence of wide variation in PU management. An understanding of current knowledge is fundamental to evidence-based practice implementation.

Methods: This was a prospective survey, using a multiple choice question format developed with nurse wound specialists. A total of 10 questions assessed PU prevention and 10 assessed management. It was distributed to nurses working at the spinal units and rehabilitation registrars. The results from the groups were analysed for similarities and differences using one- and two-way analysis of variance (ANOVA) tests and tests for significance of specific linear combinations of group means.

Results: The response rate was 79\% (19/24) and 71\% (20/28) from the two SCl units, and $46 \%$ $(13 / 28)$ from doctors. Doctors performed better than nurses on prevention questions $(P<0.005)$ but worse on management $(P<0.05)$. There was a significant difference in management knowledge $(P<0.001)$ between nurses working in the two units but not in prevention knowledge $(P<0.5)$ and interestingly years of experience did not correlate with performance $(P<0.2$ for prevention and $P<0.5$ for management questions).

Conclusions: Rehabilitation registrars score better in prevention questions, but poorer in management questions, which reflects academic rather than experiential knowledge. There are also differences in management knowledge among nurses, based on work area rather than years of experience. Although knowledge does not necessarily reflect practice, this calls for better standardisation and implementation of wound management pathways.
\end{abstract}

Spinal Cord (2012) 50, 159-164; doi:10.1038/sc.2011.88; published online 6 September 2011

Keywords: pressure ulcers; spinal cord injury; knowledge of prevention and management of pressure ulcers; gaps in knowledge; questionnaire survey

\section{Introduction}

Pressure ulcers (PUs) are a major cause of morbidity in people with spinal cord injury (SCI), with a significant impact on a person's function, physical and mental health, finances, social relationships and quality of life. It is estimated that $80 \%$ of individuals with SCI will have a PU during their lifetime. ${ }^{1}$ In a study by McKinley et al., ${ }^{2}$ PUs were the most

Correspondence: Dr N Gupta, Spinal Cord Injuries Unit, Royal North Shore Hospital, St Leonards, New South Wales; C/O 3, John street, Concord, Sydney, New South Wales 2137, Australia.

E-mail: nidhi883@hotmail.com

Received 4 December 2010; revised 30 June 2011; accepted 19 July 2011; published online 6 September 2011 common secondary medical complications of SCI with prevalence rates ranging from $15.2 \% 1$ year after injury to $29.4 \%$ at 20 years after injury. PU and its associated complications also exert their toll on the health system, contributing a disproportionate number of bed-days and long length of stays (median of 49 days). ${ }^{3}$ In Australia, the cost of treating deep PU is estimated at between \$61230 and $\$ 100000$ per patient, with an estimated annual cost nationally of up to $\$ 350$ million. ${ }^{4-6}$

Given its clinical significance, PU prevention and management should be core knowledge for all health professionals. While some studies have demonstrated a good level of 
knowledge (70-80\%) $)^{7-12}$ among nurses, others have shown limited knowledge with only $50 \%$ of nurses knowing half of the recommendations. ${ }^{13}$ This variable knowledge translates to suboptimal clinical practice, with inconsistencies in treatment and management often based on intuition, experience or habit. ${ }^{14}$ A survey in Sydney found outdated practices with regard to prevention of PUs and non-use of any assessment scales (80\%) for monitoring PUs. ${ }^{15}$ Hospitalbased education interventions have encountered limited success in reducing the occurrence of PUs. Even with sound knowledge, translation to practice can be fraught, with 1 study finding that although family physicians had reasonable knowledge $(74.4 \pm 8.1 \%)$, most of them were unaware of the Agency for Health Care Policy and Research Clinical practice guidelines on PU management. ${ }^{16}$

Attempts have been made to develop standardized PU management pathways in SCI clients by the New South Wales Statewide Spinal Cord Injury Service. A taskforce committee created a detailed, comprehensive document on PU management in 2008, which recommends pathways for patients with SCI from retrieval situations to community settings. Although comprehensive, full of useful information and evidence based, this has met with limited success in its uptake as it is lengthy, complex and sometimes repetitive. There have also been no local champions to lead enforcement of the guidelines or education of local service providers on the use of this document. The Statewide Service has also devised a simple flow diagram that advises what cases are appropriate to refer for specialised spinal plastics input. Although logical and easy to follow, it is essentially a referral guideline, not a clinical management guideline, and as such does not cover dressing types or identify cases, which need antibiotics/surgical debridement while awaiting spinal plastics input, which can have a protracted waiting list. In addition, the NSW Department of Health has policies and procedures for PU prevention, management and surveillance (reporting) of PU, but these are not specific to the SCI population. There is such a wide range of dressing materials available in the market and variability in the advice of specialist clinicians that it has been difficult to develop a common treatment pathway statewide and it is, therefore, not uncommon for different SCI units in Australia to be using different guidelines. There have also been challenges in hospitals without a spinal unit in following the guidelines due to a variety of reasons, including lack of time, inadequate skills and knowledge, inadequate staffing and unfamiliarity with the care of SCI patients (because of the relative infrequency of admissions to these hospitals).

Successful implementation of an evidence-based algorithm for PU management relies on identifying and understanding discrepancies in local knowledge and practice. As such, our aims were as follows:

(1) To assess whether there is a significant difference between two metropolitan SCI units with respect to nurses knowledge of pressure ulcer prevention and management.

(2) To assess whether knowledge varies with years of experience in nurses.
(3) To assess whether there is a significant difference in knowledge between rehabilitation registrars (training specialists) and nurses.

\section{Patients and methods}

A prospective survey was carried out between May 2009-June 2009, targeting a cluster sample of Registered and Endorsed Enrolled Nurses working on a permanent basis at two metropolitan SCI units (Unit A and B) and doctors training to specialise in rehabilitation medicine. The casemix of patients at Unit A consists predominantly of acute SCI admissions, SCI patients waiting for transfer to Unit B and chronic SCI patients who have been admitted for medical complications. Unit B's casemix predominantly consists of patients who have recently sustained an acute SCI and have been admitted for ongoing inpatient rehabilitation, with a small proportion of chronic SCI patients admitted from the community for multidisciplinary assessment. Both units would have to manage patients with PUs on a regular basis.

A 24-item questionnaire was developed by the researchers in consultation with spinal medicine specialists and clinical nurse consultants from both units. The questionnaire had three sections: the first four questions gathered demographic information (for example, years of experience, training and scale used), the next 10 multiple choice questions assessed prevention and the last 10 multiple choice questions focused on management. There were no open-ended questions in the 20 questions with four possible choices (A-D) for each. For each question, if the respondent answered correctly, a 'yes' value was recorded, otherwise a 'no' value was recorded. The data were converted to numerical format for analysis by counting the number of 'yes' values by question and/or respondent. We also looked at the variation of choices for each question (that is, we looked to see if respondents had been choosing an alternative answer more frequently as a group). De-identified photographs were utilised in the management section.

Bundles of numbered questionnaires containing an information sheet and consent form were given to nursing unit managers on the two units, who facilitated distribution to all permanent full and part time nursing staff. There were 28 nurses in Unit A and 24 in Unit B who met the criteria. Participation was voluntary and we did not remunerate people for their time. The questionnaires did not request any identifying details such as name, age or date of birth as we felt that the anonymity might increase the response rate. Rehabilitation medicine trainees were contacted through the Faculty of Rehabilitation Medicine's education coordinator for the state and copies distributed to the 28 registrars present at a monthly teaching session. Completed copies were collected from the units or doctors personally, or were received through post. Various local methods were used with the assistance of nursing unit managers and the Australasian college of Rehabilitation Medicine to enhance the response rate.

Approval for the study was given by the local Health Service Human Research Ethics Committee. We certify that all applicable institutional and governmental regulations 
concerning the ethical use of human volunteers were followed during the course of this research.

Statistical analysis

The software package $\mathrm{R}$ version 2.8. 1 was used for all statistical analyses (available on http://www.r-project.org). Separate one-way analysis of variance (ANOVA) tests were used to detect differences in average scores by work area (Units A, B and doctors), years of experience and job type (doctor/nurse). A two-way ANOVA test was used to test for significant differences in mean scores in a model including both work area and job type. Tests for linear contrasts were used to test for significant differences in average scores between the two nursing units. We undertook analysis of the two sections of the questionnaire collectively and by sections of prevention (10 multiple choice questions) and treatment (10 multiple choice questions).

\section{Results}

\section{Background demographics}

There were 20/28 respondents from Unit A (71\%), 19/24 respondents from Unit B (79\%) and 13/28 respondents from rehabilitation registrars (46\%). Unit A had a higher percentage of nurses with 1-5 years experience, whereas Unit B had a higher percentage of those with $10+$ years of experience (Table 1). Rehabilitation registrars are rotated through spinal units for 6 months over a 4-year full time training programme, hence the doctors had either 6 months or no experience in SCI management. Of the doctors who responded, 7 had not worked in a SCI unit, whereas 6 had 6 months of work experience in a SCI unit.

Total scores (assessment and management questions combined) The average score of the three groups combined was $6.44 / 10$ for the assessment/prevention questions and 5.92/10 for the management questions, with a total average score of 12.38 out of a possible 20 .

Job type. There was no significant difference in overall mean scores between doctors $($ mean $=12.54$; s.d. $=2.60$ ) and nurses combined (mean $=12.33$; s.d. $=2.76$; one-way ANOVA, $P>0.5)$.

Work area. Unit A had the highest average overall score $($ mean $=13.15$; s.d. $=2.72)$, followed by doctors $($ mean $=$ 12.54 ; s.d. $=2.60)$ and unit $\mathrm{B}($ mean $=11.47$, s.d. $=2.59$; Figure 1). However, the average scores were not significantly different between the three work area groups (one-way ANOVA, $0.1<P<0.2$ ).

Table 1 Distribution of years of experience by the nursing group

\begin{tabular}{lcc}
\hline & Unit $A(\%)$ & Unit B (\%) \\
\hline$<1$ year & 5 & 5 \\
$1-5$ years & 35 & 11 \\
$5-10$ years & 20 & 21 \\
$>10$ years & 40 & 63 \\
\hline
\end{tabular}

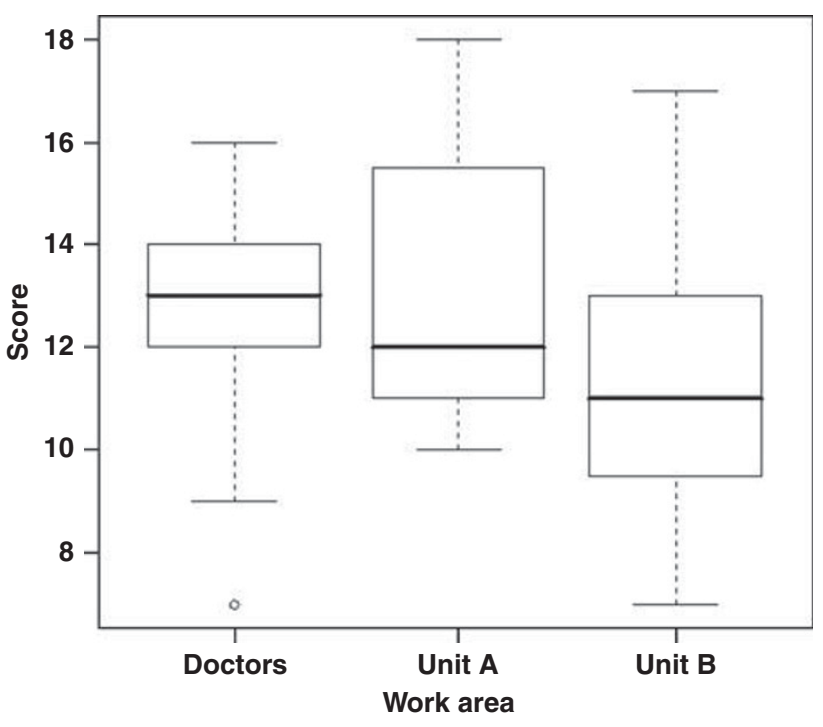

Figure 1 Total scores by work area.

Years of experience in nurses. Among nurses, those with $>10$ years of training had the highest average scores (mean $=13.15$; s.d. $=2.72$ ). However, there was no significant difference in average overall scores between different levels of experience in nurses (one-way ANOVA, $0.1<P<0.2)$.

\section{Prevention questions}

The average score for prevention questions for all respondents was 6.44/10. The poorest performing question (mean $36.9 \%$; range $25-47.4 \%$ ) asked how long the pressurerelieving manoeuvre should be. The best performing question (mean 95.8\%; range 92.3-100\%) asked how quickly after exposure it took for a PU to develop.

Job type (doctors versus nurses). The average score for doctors (mean $=7.69$; s.d. $=1.11$ ) was greater than the average score for nurses $($ mean $=6.03$; s.d. $=1.71)$, a difference that was statistically significant (one-way ANOVA, $P<0.005$ ).

Work area. Rehabilitation medicine registrars had the highest average score for prevention questions (mean $=7.69$; s.d. $=1.11)$, followed by unit $B($ mean $=6.32$; s.d. $=1.70)$ and unit A (mean $=5.75$, s.d. $=1.71$; Figure 2$)$. A one-way ANOVA test by work area was significant $(P<0.005)$. However, this was due to differences between doctors and nurses (see above), as there was no statistically significant difference in performance between the nurses in units $\mathrm{A}$ and $\mathrm{B}$ $(0.2<P<0.5)$.

Years of experience in nurses. There was no significant difference based on years of experience among nurses for prevention questions (one-way ANOVA, $(0.1<P<0.2)$.

\section{Management questions}

The average score for management questions for all respondents was 5.92/10. The poorest performing question (mean $20.4 \%$; range $0-35 \%$ ) asked for the most appropriate dressing 


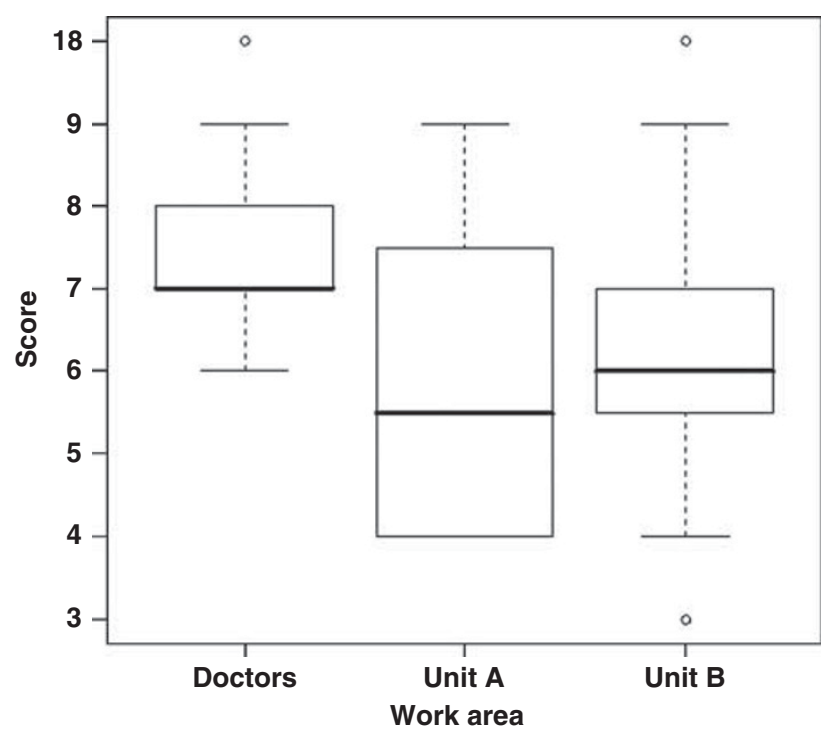

Figure 2 Prevention scores by work area.

type for a minimally exudative heel wound. The other question (mean 20.5\%; range 15.4-25\%) that performed poorly related to a sacral PU, asking for the next most appropriate step if no progress was seen with dressings. The best performing question (mean 97.4\%; range 92.3-100\%) asked for the most appropriate advice when a patient is found with a new PU.

Job type (doctors versus nurses). The average score for nurses (mean $=6.28$; s.d. $=2.15)$ was significantly greater than the average score for doctors (mean $=4.85$; s.d. $=2.15$; one-way ANOVA, $0.01<P<0.05)$.

Work area. Unit A had the highest average score for management questions $($ mean $=7.35$; s.d. $=1.39$ ), followed by Unit B $($ mean $=5.16 ;$ s.d. $=2.27)$ and doctors (mean $=4.85$; s.d. $=2.15$; Figure 3 ). A one-way ANOVA test found work area to be strongly significant $(P$-value $<0.001)$. Further tests showed a significant difference between Unit A and $\mathrm{B}(P<0.001)$.

Years of experience in nurses. Nurses with 1-5 years of experience had the highest average scores (mean $=6.78$; s.d. $=2.22$ ) closely followed by nurses with $10+$ years of experience $($ mean $=6.45$; s.d. $=2.31)$. Nurses with $<1$ year experience scored the lowest $($ mean $=4.50 ;$ s.d. $=2.04)$. There was no significant difference in management test scores by different levels of experience among nurses (oneway ANOVA, $0.2<P$-value $<0.5)$.

\section{Discussion}

To our knowledge, this is the first three-way comparison study between health professionals involved in the care of people with SCI. Although this was a small study, our response rates were good (71-79\% among nurses and $46 \%$ in

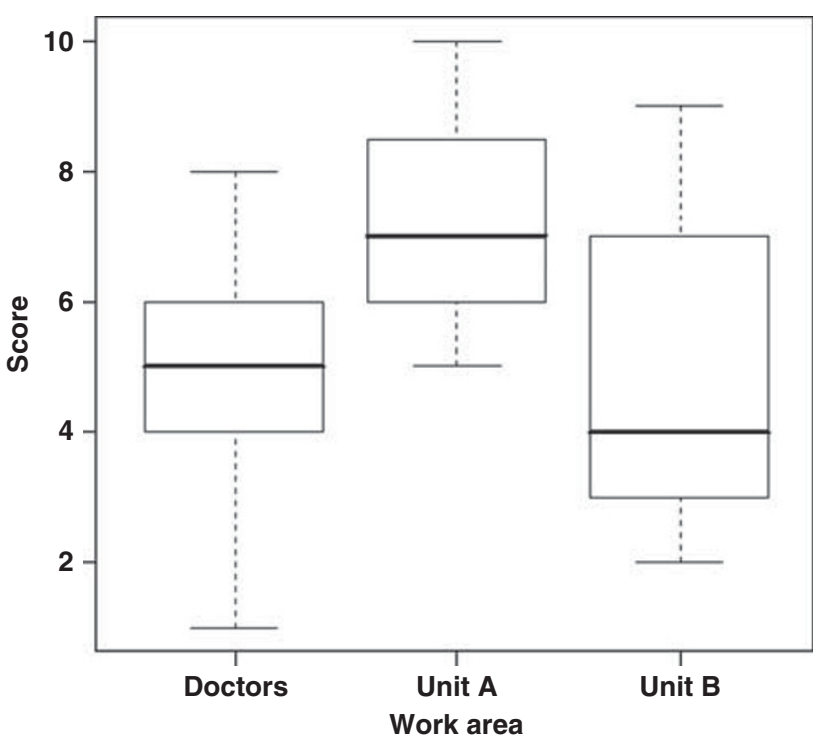

Figure 3 Management scores by work area.

doctors), compared with other studies utilising similar questionnaire designs, which range from $25-35 \% .{ }^{14,15}$ Response bias was minimised by voluntary participation and by removal of identifying information. Furthermore, the use of nursing unit managers to facilitate distribution of the surveys assisted us to target a representative sample of the population of interest.

\section{Assessment and prevention knowledge}

The NSW Department of Health has issued policies that mandate that each health service must have a PU prevention programme that contains as a minimum standard the following elements: a standardised PU risk assessment tool, best practice guidelines for the prevention of PUs, an education programme on PU prevention, a system in place for easy access to PU prevention resources, a PU reporting system and a local governance system to monitor the effectiveness of the PU prevention programme. ${ }^{17}$ The wording is general and leaves it up to the health service to implement specifics. In addition, there are wound assessment, wound care and management guidelines available on the NSW Health intranet site. ${ }^{18}$ Unit A has adapted one of these guidelines ${ }^{19}$ for the nursing care of patients with SCI, such as a lift round every 3 hours (for skin checks and repositioning), ensuring every patient has a care plan (which includes the Waterlow risk assessment scale) and adopting standardised initial actions if a PU is noticed (remove pressure off a grade $1 \mathrm{PU}$ and if it is a grade 2 or worse PU; request a decision on treatment from a senior nurse). The Waterlow risk assessment scale is based on eight variables (weight and build, continence, skin type, mobility, gender and age, appetite with a special section incorporating tissue malnutrition, neurological deficits, surgery/trauma and special medication). Scores are totalled to produce a summary score from 3 (best prognosis) to 45 (worst prognosis). ${ }^{20}$ The patient's care plan is revised on a weekly basis. 
Unit B has a slightly different guideline in place, which has elements from Department of Health guidelines, Australian Woundcare Association and Paralysed Veterans Association (Spinal Cord Consortium) guidelines. ${ }^{18,21,22}$ Patients are scored on the Braden scale on admission, monthly or when a PU has developed. The frequency of skin checks is also different, with Unit B doing 2 hourly skin checks on admission for the first night and then individualising the frequency according to their skin, mattress trials and client comfort. The Braden scale is a $6-23$ point scale, which evaluates skin breakdown in six domains: (sensory perception, moisture, activity, mobility, nutrition, and friction and shear). Higher scores are equivalent to better prognosis. Cutoff scores $\leqslant 10$ have been suggested as scores indicative of those who develop PU in individuals with SCI. ${ }^{20}$ We are unsure of the history of the choice of risk assessment scales in either unit. To our knowledge, the Braden scale has the best combined validity and utility evidence though more specific testing in SCI population is required. ${ }^{20,23,24}$ Neither of the scales demonstrates excellent validity. ${ }^{20}$ There is also only level 4 evidence supporting the use of validated risk assessment tools for the prevention of PU even in the general population. $^{25}$

We found that $90 \%(18 / 20)$ of nurses in Unit A reported using the Waterlow scale, compared with $73.7 \%(14 / 19)$ in Unit B for the Braden scale. Overall, 61.5\% (8/13) of doctors were familiar with the Braden and/or Waterlow scales. The lower utilisation rate in Unit B and in doctors could reflect lack of emphasis or education on the tools, dissatisfaction with tools chosen or uncertainty with their use. Although both spinal units have experienced nurses who utilise the care plans and guidelines, both have a proportion of casual nursing staff who may not be aware of the procedure or who do not inform the nursing staff of changes in the clients' skin integrity.

When asked to stage a PU from a photograph, $45 \%$ of Unit A, $31 \%$ of Unit B and $61.5 \%$ of doctors had correct responses. Staging a PU is important for ascertaining the extent of damage and in NSW, forms part of a statewide algorithm for PU management. The low performance in this area highlights a major gap in knowledge that needs to be addressed in future education and incorporated into ward practice.

Respondents uniformly scored poorly (mean 36.9\%) for the question 'how long should a pressure-relieving manoeuvre be?' We consider this core knowledge, something that should be conveyed to patients consistently and regularly. Apart from this question, doctors scored fairly well on the other nine questions (61.5-100\%). The other area of poor performance among nurses was frequency of pressure relief (Unit A mean 15.8\%, Unit B mean 36.8\%). There was no significant overall difference in scores between units or by years of experience.

\section{Management knowledge}

Although doctors scored better than nurses in prevention, the opposite was found in management. This reflects the often academic rather than experiential knowledge of trainees. Trainees often see wound management as the domain of nurses and the plastics surgery specialty. They also have little 'hands-on' knowledge of dressing materials, given the vast variety available and find it confusing when asked to select which materials to use on different wounds. The lack of easily available standardised protocols and conclusive evidence-based reviews on dressing management may also have bearing on this gap in knowledge and confusion. Involving trainees in day-to-day wound management is thus essential to improve their practical knowledge.

Work area was a significant variant as well, with Unit A having a significantly higher mean score than Unit B. In terms of casemix, Unit A has a larger proportion of patients with complex PUs and this exposure could, therefore, increase knowledge and confidence in management, reflected in the better scores. Specifically, Unit A performed significantly better than Unit B on 4 out of the 10 management questions (q 16, q 17, q 20 and q 24). However, it is not uncommon for patients to develop PUs while in Unit B. Furthermore, it is in rehabilitation units, that patients acquire the necessary skills, knowledge and attitudes that will set them up for successful community integration, of which good skin care is integral and paramount. Although Unit $B$ has the role of prevention education, it did not score significantly better than Unit A, which is an interesting observation. The discrepancy in knowledge may reflect different practices between the two units, which may be a concern as the same group of patients are often managed at the two units at different times. Further testing across all three groups was conducted to rule out cultural bias between groups as a reason for the significant results.

Previous studies have found no variation with work area but significant variation with years of experience. ${ }^{14,26}$ In our study, we did not find a correlation between years of experience and higher scores, which was surprising as we hypothesised that years of experience might relate to more training. However, we were unable to assess for qualification and training variants as outcome measures as most staff had multiple types of training experience and the information to weight each class of training was not available. Therefore, no definite conclusions could be drawn from our study on the influence of training type on test performance. The insignificance of years of experience strengthens the argument that work area is the primary driver of PU prevention and management and where efforts should be directed to improve patient outcomes.

Utilising self-administered questionnaires to test knowledge has its limitations, especially as respondents might confer with each other or refer to online/textbook sources. However, if this had been the case, we would have expected a higher score especially in prevention questions as the answers are easily available. There were also no identical responses, which indicates that there was no verbatim copying of answers (group responses). To a certain extent, the anonymity may have helped respondents answer without fear of embarrassment. Although our sample size was small, we had a reasonable response rate from nurses in Unit $\mathrm{A}$ and $\mathrm{B}$. However, the response rate from doctors was lower 
(46\%), and the doctors that participated were mostly senior trainees (further advanced into their 4 years of specialist training). This might have biased the results towards a higher mean score among doctors, and therefore their results should be interpreted with caution. If more junior trainees had participated, this might have resulted in a smaller or less significant difference between doctors and nurses in prevention, but an increased difference in the management section, which may add strength to our study findings. This study highlights deficits in knowledge among a highly specialized cohort of health care professionals on PU care. Development of national and local care pathways for their management has been attempted in the past but has not been implemented in units due to multiple factors previously discussed. Prevention regimes in different phases of SCI have been noted to be different, which can be confusing. Simple tables showing the prevention regimes in the different stages of SCI would be one-way of addressing the problem. Development of standardized flow diagrams for all levels of care and dressing regimes for various types of PUs in SCI may also help. Fostering interunit collaboration and rotations (some units have agreements that clinicians can rotate between community and inpatient settings to get a broader range of experience), regular chart audits on management, agreement on best risk assessment scales and evidence-based guidelines to use, finding clinical champions and having adequate funding to support implementation are also fundamental.

Further study is required into attitudes of health care professionals, ward admission procedures/protocols and training provided to identify possible barriers to effective PU prevention and management and avenues to improve our organisational practice.

\section{Conclusion}

Our study has shown significant gaps in core knowledge in areas of prevention and management in health professionals caring for people with SCI. Doctors scored better than nurses in prevention, but did significantly worse in terms of management. There are differences between units in knowledge and perhaps practice. This highlights a need for an increased focus on PU education, standardisation and implementation of wound management pathways.

\section{Conflict of interest}

The authors declare no conflict of interest.

\section{Acknowledgements}

We thank the study participants for giving their time so generously. We also acknowledge the clinical nurse consultants, the nursing unit managers and the Faculty of Rehabilitation Medicine who helped with the questionnaire design and distribution and collection of completed surveys.

\section{References}

1 Prevention of Pressure Sores through skincare SCI InfoSheets available online http://www.spinalcord.uab.edu/show.asp?durki= 21479 (accessed 19 August 2011).

2 McKinley WO, Jackson B, Cardenas DD, DeVivo MJ. Long-term medical complications after traumatic spinal cord injury: a regional model systems analysis. Arch Phys Med Rehabil 1999; 80: 1402-1410.

3 Middleton JW, Lim K, Taylor L, Soden R, Rutkowski S. Patterns of morbidity and rehospitalisation following spinal cord injury. Spinal Cord 2004; 42: 359-367.

4 Roland J. Pressure ulcers:a literature review and a treatment scheme. Aust Fam Physician 1993; 22: 1819-1827.

5 Young C. What cost a pressure ulcer? Prim Intent 1997; November 24-31.

6 Klei M, Maclellan L, Maclellan D. Bottoms up:avoiding the horrors of pressure ulcers. Vetrans' Health 1997; 20: 24-27.

7 Bostrom J, Kenneth H. Staff nurse knowledge and perceptions about prevention of pressure sores. Dermat Nurs 1992; 4: 365-367.

8 Beitz JM, Fey J. Perceived need for education vs. actual knowledge of pressure ulcer care in a hospital nursing staff. Med Surg Nurs 1998; 7: 293-301.

9 Provo B, Piacentine L, Dean-Barr S. Practice versus knowledge when it comes to pressure ulcer prevention. I Wound Ostomy Continence Nurs 1997; 24: 265-269.

10 Russell L. Knowledge and practice en pressure area care. Prof Nurse 1996; 11: 301-306.

11 Anthony D, Mockridge J. Nurses' knowledge about pressure sore treatment and healing. Nurs Stand 1999; 13: 68-71.

12 Källman U, Suserud BO. Knowledge, attitudes and practice among nursing staff concerning pressure ulcer prevention and treatment - a survey in a Swedish healthcare setting. Scand J Caring Sci 2009; 23: 334-341.

13 Egglink M, Halfens RJ. Knowledge, beliefs and use of nursing methods in preventing pressure sores in Dutch hospitals. Int J Nurs Stud 1995; 32: 16-26.

14 Pedro Luis PH, Francisco Pedro G. Pressure ulcer care in Spain: nurses' knowledge and clinical practice. J Adv Nurs 2007; 58: 327-338.

15 Sharp K, Burr G, Broadbent M, Cummins M. Pressure ulcer prevention and care: a survey of current practice. J Qual Clin Pract 2000; 20: 150-157.

16 Kimura S, Pacala J. Pressure ulcers in adults: family physicians knowledge, attitudes, practice, preferences and awareness of AHCPR guidelines. J Fam Pract 1997; 44: 361-368.

17 Clinical practices Pressure ulcer prevention document available from http://www.health.nsw.gov.au/policies/PD/2005/PD2005_ 257.html (accessed 19 August 2011).

18 www.health.nsw.gov.au/resources/nursing/practioner/cpg_wound_ mment_hneahs_pdf.asp (accessed 20 August 2011).

19 NSCCAHS wound assessment guideline GE2008_034 available from http://www.nscchealth.nsw.gov.au/services/wound.care/ woundassessment.pdf (accessed 19 August 2011).

20 Mortwnson WB, Millwe W. A review of scales for assessing the risk of developing a pressure ulcer in individuals with SCI. Spinal Cord 2008; 46: 168-175.

21 CSCM (2000) Pressure Ulcer Prevention and Treatment following Spinal Cord Injury:A Clinical Practice Guideline for Health Care Professionals [Online]. Available from http://www.pva.org/site/c.ajIRK9 NJLcJ2E/b.6305401/k.BCBB/Home.htm (accessed 20 August 2011).

22 Assessed from Royal Rehabilitation Centre Sydney, Centre Manual Policy and Procedure, Skin Integrity:Wound Management (accessible through RRCS intranet website only) (accessed 15 February 2011).

23 Salzberg AC, Byrne D, Kabir R, Niewerburg P, Cayten CG. Predicting pressure ulcers during initial hospitalization for acute spinal cord injury. Wounds 1999; 11: 45-57.

24 Wellard S, Lo SK. Comparing Norton, Braden and Waterlow risk assessment scales for pressure ulcers in spinal cord injuries. Contemp Nurse 2000; 9: 155-1690.

25 Keast D, Parslow N, Houghton P, Norton L, Fraser C. Best practices for the prevention and treatment of pressure ulcers: update 2006. Wound Care Can 2006; 4: 31-43.

26 Pieper B, Mott M. Nurses' knowledge of pressure ulcer prevention, staging, and description. Adv Wound Care 1995; 8: 34-48. 